\title{
Observations Of General Learning Patterns In An Upper- Level Thermal Physics Course
}

\author{
David E. Meltzer
}

College of Teacher Education and Leadership, Arizona State University, Polytechnic Campus, Mesa, AZ 85212, USA

\begin{abstract}
I discuss some observations from using interactive-engagement instructional methods in an upper-level thermal physics course over a two-year period. From the standpoint of the subject matter knowledge of the upper-level students, there was a striking persistence of common learning difficulties previously observed in students enrolled in the introductory course, accompanied, however, by some notable contrasts between the groups. More broadly, I comment on comparisons and contrasts regarding general pedagogical issues among different student sub-populations, for example: differences in the receptivity of lower- and upper-level students to diagrammatic representations; varying receptivity to tutorial-style instructional approach within the upper-level population; and contrasting approaches to learning among physics and engineering sub-populations in the upper-level course with regard to use of symbolic notation, mathematical equations, and readiness to employ verbal explanations.
\end{abstract}

Keywords: physics education, thermodynamics.

PACS: 01.30.Cc; 01.40.Fk 
The set of written questions (along with other questions) was administered on the first day of class to a total of 33 students enrolled in the upper-level thermal physics course during 2003 and 2004. Two of the questions that were administered to all of the students are shown in Figure 1. The first question may be answered by examining the area under the curves representing Process \#1 and Process \#2, respectively. Since the area under the curve representing Process \#1 is the larger, the work done by the system during Process \#1 is greater than that done during Process \#2. We found that a significant proportion of students in all samples responded by claiming that the work done by the system in the two processes was the same $\left(W_{1}=W_{2}\right)$. For instance, this response was given by $21 \%$ of the students in the thermal physics course; see Table 1. (Note that 2003 and 2004 results have been combined since they are very similar to each other.) Examples of explanations offered by students include: "Equal, path independent," and "Equal, the work is the same regardless of path taken.”

A correct response to the second question required some understanding of the first law of thermodynamics. Since the change in internal energy is the same in both processes but more work is done by the system in Process \#1, the system must absorb more energy in the form of heat in Process \#1 in order to reach the same final state (so we will have $Q_{1}>Q_{2}$ ).

If one considers only correct answers that are also accompanied by correct or partially correct explanations, the thermal physics students had superior
This $P-V$ diagram represents a system consisting of a fixed amount of ideal gas that undergoes two different processes in going from state A to state B:

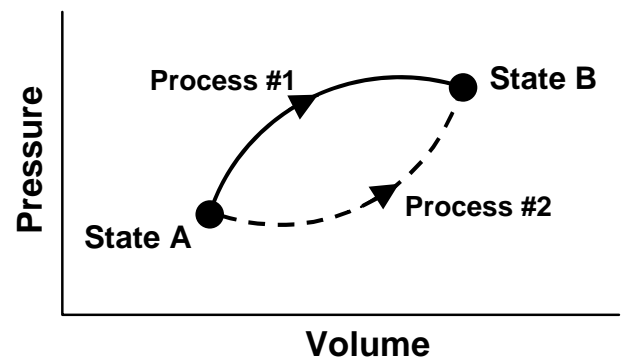

[In these questions, $\boldsymbol{W}$ represents the work done $\boldsymbol{b y}$ the system during a process; $\boldsymbol{Q}$ represents the heat absorbed by the system during a process.]

1. Is $W$ for Process \#1 greater than, less than, or equal to that for Process \#2? Explain.

2. Is $Q$ for Process \#1 greater than, less than, or equal to that for Process \#2? Please explain your answer.

FIGURE 1. Two of the questions posed to students in both introductory and upper-level physics courses. Answers: (1) greater than; (2) greater than.

TABLE 1. Responses to Diagnostic Questions \#1 and \#2.

\begin{tabular}{|c|c|c|c|}
\hline & $\begin{array}{c}\text { 1999-2001 } \\
\text { Introductory Physics } \\
\text { Written Sample } \\
\text { (Post-test) } \\
N=653\end{array}$ & $\begin{array}{c}2002 \\
\text { Introductory Physics } \\
\text { Interview Sample } \\
\text { (Post-test) } \\
N=32\end{array}$ & $\begin{array}{c}\text { 2003-2004 } \\
\text { Thermal } \\
\text { Physics } \\
\text { (Pretest) } \\
N=33\end{array}$ \\
\hline$W_{1}=W_{2}$ [incorrect $]$ & $30 \%$ & $22 \%$ & $21 \%$ \\
\hline$Q_{1}>Q_{2}$ & $45 \%$ & $34 \%$ & $33 \%$ \\
\hline $\begin{array}{l}\text { Adequate explanation } \\
\text { (Correct or partially correct) }\end{array}$ & $11 \%$ & $19 \%$ & $30 \%$ \\
\hline
\end{tabular}

performance compared to the broader sample of introductory students. However, the difference in performance between the thermal physics students and the interview sample is not statistically significant. In any case, a correct-response rate of less than one-third would probably not be considered adequate by most instructors for a group that is supposed to be beginning study of statistical mechanics.
In analogy to the explanations offered for Question \#1, the most popular incorrect response on Question \#2 was that $Q_{1}=Q_{2}$, and the most popular explanation for that answer was that the heat transfer to a system during a process was independent of the path taken by the system during the process. Since the initial and final states were the same, many students argued, the heat absorbed also had to be the same. Thus we found 
that many students at both the introductory and advanced level used similar incorrect arguments for both problems.

At the beginning of the thermal physics course in 2004, students were given the opportunity to respond to an additional set of questions that had been answered by the interview sample in the introductory course. These included questions regarding an idealgas system that undergoes a cyclic process employing an isobaric expansion and an isothermal compression. Our results demonstrated quite consistently that most of the upper-level students retained serious conceptual difficulties regarding fundamental concepts, including the following: (1) Most students believed that, over the course of the cyclic process, the net work done by the system and the net heat transfer to the system had to equal zero; (2) most students did not realize that heat transfer away from the gas system had to occur during the isothermal compression; (3) about a quarter of the students believed that the total kinetic energy of the ideal-gas molecules would have to increase during an isothermal compression; (4) more than one third of the students believed that positive work is done on the system by the surrounding environment during an isobaric expansion.

In fact, there was little or no significant difference in performance between upper-level students and those in the interview sample on most questions, consistent with the data shown in Table 1 . This suggests that a significant proportion of upper-division students beginning advanced study of thermodynamics are still struggling with fundamental concepts of heat, work, and the first law of thermodynamics that are normally presumed to have been mastered in their introductory courses.

We also explored students' understanding of the second law of thermodynamics and the principle of entropy increase during spontaneous processes. The results show both similarities and differences between the introductory and upper-level students. The introductory students have a tendency to argue that the "system entropy" must always increase, even in cases where inadequate information is available to make such a determination. At the same time, these students are slow to accept the idea that the total entropy of system and surroundings must increase during naturally occurring ("spontaneous") processes [4]. In contrast to the introductory students, the students in the thermal physics course readily accept the principle that total entropy increases in naturally occurring processes. However, they share with the introductory students the tendency to assume that "system entropy" must always increase regardless of process and regardless of how the "system" is defined. Nonetheless, in comparison to the introductory students, a significantly larger proportion of upper-level students gave consistently correct responses on the entropychange questions. This suggests that upper-level students have a distinctive pattern of thinking that retains many similarities to introductory students and yet is not identical to it.

\section{UPPER-LEVEL STUDENTS DEMONSTRATED A SUPERIOR BUT UNEVEN GRASP OF QUALITATIVE REASONING SKILLS}

Students in the upper-level course demonstrated a number of important learning skills that were significantly better developed than among students in the introductory course. At the same time, even very able students in the advanced course periodically demonstrated a vulnerability to learning difficulties similar or identical to those found among students in the introductory course.

Upper-level students demonstrated an ability to make use of qualitative reasoning, multiple representations, and guided-inquiry curricular materials that was generally beyond that of the introductory students. In covering similar material, upper-level students were quicker to generalize over specific contexts with a unifying concept. By contrast, introductory students tended to focus on pattern matching, recognizing commonalities among different problems without necessarily extracting a unifying physical theme. Despite having superior mathematical skills, upper-level students relied less on purely mathematical calculations and arguments than did introductory students in working identical problems. They were less likely to simply point to an equation as an explanation, and more likely to use arguments based on proportional reasoning.

Upper-level students found it easier than did introductory students to interpret the meaning of diagrams, bar charts, and other graphical material. They were more comfortable in making use of multiple representations (verbal, diagrammatic, etc.) to express their own thinking, and they showed less reliance on purely mathematical forms of reasoning. However, although upper-level students made more effective use of qualitative arguments when dealing with relatively familiar concepts, when dealing with less familiar problems they tended to rely on their relatively advanced mathematics skills and used more algebraic and quantitative arguments, sometimes successfully, and sometimes not. Moreover, their readiness to use qualitative techniques (such as $\mathrm{P}-\mathrm{V}$ 
diagrams) still fell short of what experts would consider desirable.

Upper-level students made effective use of guidedinquiry worksheets originally developed for use with introductory students. They required less guidance from instructors, in part because they were less likely to become bogged down in problem minutiae such as instructions or descriptions of apparatus, and showed less confusion in interpreting instructions.

\section{SUBJECTIVE ASPECTS}

Consistent with observations made among students in introductory courses, both highly favorable and highly unfavorable reactions toward interactiveengagement techniques were displayed by upper-level students. The $10-30 \%$ unfavorable rating on evaluations covered a broader range than found in the introductory algebra-based course; both highly favorable and highly unfavorable opinions co-existed in the same class. Use of guided-inquiry worksheets during class (instead of in a separate recitation section) created logistical difficulties due to the broad range of speeds with which students worked. Insufficient pretesting and lack of previous relevant research made optimal course planning difficult.

\section{ISSUES ASSOCIATED WITH ENGINEERING STUDENTS}

Although there were some notable exceptions, it seemed that the majority of the engineering students (and physics-engineering double majors) were relatively less familiar or comfortable with the course requirement that they provide explanations for reasoning in problem-solving, in comparison to the majority of the physics majors. There seemed to be a greater tendency to favor methods (sometimes called "plug-and-chug”) of simply substituting numbers into equations without providing required explanations of reasoning. Some students attempted to defend this process as being the more appropriate method for an upper-level science course, despite the fact that the course instructor had made very clear that expectations were different for this particular class. Some students demonstrated a persistent and inappropriate tendency to employ notations and specific algebraic expressions learned in engineering courses, even when they had been pulled out of context and therefore conflicted directly with those used in the thermal physics course. (This sometimes extended to an unwillingness to express algebraic answers using the symbols employed in the text, notes, lectures and homework assignments of the thermal physics course.) In general, overt expressions of dissatisfaction with the course and the instructional methods (though not necessarily dissatisfaction itself) seemed more common among the engineering students. To be fair, our sample of such students was quite small, there were some very notable exceptions to the general pattern, and we certainly draw no implication that the students were accurately representing the intent of the instructional methods in upper-level engineering courses.

\section{METHODOLOGICAL ISSUES}

Our experience in this course emphasized a need to take into account the different backgrounds and notational conventions of engineering students when analyzing, interpreting, and categorizing their responses to diagnostic questions. Our difficulty in following students' chains of reasoning was often increased by their adherence to non-standard (from the physics standpoint) notations and lines of argument.

\section{ACKNOWLEDGMENTS}

This work has been supported in part by NSF DUE-9981140 [T. J. Greenbowe, Co-Principal Investigator], PHY-0406724, PHY-0604703 [M. McDermott, Principal Investigator], and DUE-0817282 [J. Thompson, Principal Investigator]. Warren Christensen and the late Ngoc-Loan Nguyen contributed significantly to the research reported here.

\section{REFERENCES}

1. D. E. Meltzer, Am. J. Phys. 72, 1432-1446 (2004).

2. D. E. Meltzer, "Student Learning in Upper-Level Thermal Physics: Comparisons and Contrasts with Students in Introductory Courses” in 2004 Physics Education Research Conference [Sacramento, California, 4-5 August 2004], edited by J. Marx, P. R. L. Heron, and S. Franklin, AIP Conference Proceedings 790, American Institute of Physics, Melville, NY, 2004, pp. 31-34; D. E. Meltzer, “Investigation of student learning in thermodynamics and implications for instruction in chemistry and engineering," in 2006 Physics Education Research Conference [Syracuse, New York, 26-27 July 2006], edited by Laura McCullough, Leon Hsu, and Paula Heron, AIP Conference Proceedings 883, American Institute of Physics, Melville, NY, 2007, pp. 38-41.

3. M. E. Loverude, C. H. Kautz, and P. R. L. Heron, Am. J. Phys. 70, 137-148 (2002); M. J. Cochran and P. R. L. Heron, Am. J. Phys. 74, 734-741 (2006).

4. W. Christensen, D. E. Meltzer, and C. A. Ogilvie, Am. J. Phys., in press. 